\title{
Energy Accumulation and Dissipation Analysis of Simply Supported Concrete Beams under Uniform Loading
}

\author{
Chunliu Li ${ }^{1,2, *}$, Shuangjun $\mathrm{Xu}^{1}$, Hao Guo ${ }^{1}$, Yan Zhao ${ }^{1}$ and Yubo Chen ${ }^{2}$ \\ ${ }^{1}$ Institute of Urban Construction, Hebei Normal University of Science \& Technology, Qinhuangdao 066004, China \\ ${ }^{2}$ International Joint Research Laboratory of Henan Province for Underground Space Development and Disaster Prevention, \\ Henan Polytechnic University, Jiaozuo 454003, China
}

Received 29 July 2021; Accepted 15 November 2021

\begin{abstract}
Due to insufficient understanding of energy accumulation and dissipation characteristics of the bending beam, the instability mechanism of concrete beam driven by the energy release has become a bottleneck problem affecting structure safety. The bending deformation behaviors of concrete beams under external loads are always accompanied by energy accumulation and release, taking simply supported concrete beams under uniformly distributed loading as research object, the structural mechanics model of concrete beam was constructed based on the energy principle, the characteristics of strain energy accumulation and dissipation of the loaded concrete beams were analyzed. For the C30 concrete, the corresponding numerical simulation was carried out, the strain energy distribution and failure characteristics of the beam structures under uniformly distributed loads were obtained. Results show that the bridge type distribution of the strain energy occurs in the simply supported concrete beams after bending under uniform loading. The failure units of the simply supported beam are concentrated in the releasing zone of the strain energy. The dissipated energy of the tensile-shear failure zone is higher than that of the shear failure zone, and the dissipated energy at bottom of the beam is significantly higher than that of other layers. The conclusions obtained in this study provide the reference for the energy evolution analysis of the loaded concrete beams.
\end{abstract}

Keywords: Uniform load, Concrete beam, Strain energy, Energy dissipation

\section{Introduction}

The simply supported concrete beams are widely used in building structures, their damage and instability directly affect the safety and economic benefits of project. Fracture and damage of the simply supported beam are the hot issues in the world. The damage and fracture of concrete materials under the action of external load are accompanied by the external energy input, accumulation and release. The energy evolution characteristics of different positions of the loaded concrete beams in each deformation stage have become a new problem in the study of the structure stability.

The concrete beam is a multiphase composite made of cement and various admixtures. Under the action of the external loads, the internal micro-cracks will expand and converge continuously. After reaching the critical load, the formation of macroscopic main cracks will eventually lead to the failure of the beam structure. The microscopic defects such as cracks and pores are main damage forms of the quasi-brittle materials. Due to the uneven distribution of the internal cracks of the concrete beam, the varying lengths and the mutual influence, it is very difficult to analyze the deterioration characteristics of the mechanical properties of the simply supported concrete beams from the initiation and development process of the material microscopic defects. So, the instability mechanism has become a bottleneck restricting the safety control of building structures.

The damage of the simply supported concrete beams has various forms and changes, but they all have a common

*E-mail address: Iclc_010@163.com

ISSN: 1791-2377 @ 2021 School of Science, IHU. All rights reserved.

doi:10.25103/jestr.145.07 feature, that is, they are all irreversible processes requiring energy dissipation [1-2]. Judging the cracking process of concrete from the perspective of energy balance, avoiding the stress field analysis at the crack tip, judging the stability of the crack based on the energy change of the entire system during the crack propagation process, which has more advantages than the stress-strain analysis method [3-5]. Therefore, based on the principle of energy balance in the process of instability fracture of the concrete structures, the energy accumulation, release and dissipation characteristics of the simply supported beams are analyzed, which will help to deeply reveal the fracture failure process and the instability threshold of concrete. The research on mechanical instability of the simply supported beam under uniform loading is of the important theoretical significance.

\section{State of the art}

To explore mechanical properties of the concrete beams under external loads, three-point bending tests of concrete beams under concentrated loads and the bending tests of concrete beams (slabs) under uniform loads have been carried out widely. Based on the physical model of threepoint bending tests, Tung and Tue analyzed the deformation fracture process of the three-point bending concrete specimens by numerical simulation [6]. Ghahremannejad and Abolmaai analyzed the displacement control of the concrete beams under uniformly distributed loads and studied the ultimate shear strength of the concrete beams and the deformation characteristics at different positions of the 
beams [7]. Pham and Tan carried out a physical simulation of mechanical response of the concrete beam under static and dynamic loads, and they analyzed the entire process from the initiation expansion of concrete cracks to the macroscopic failure of the specimen [8]. The deformation failure of concrete materials will be accompanied by energy evolution behavior. Structural health monitoring tests show that the concrete beams under uniformly distributed loads have the energy accumulation behavior, but the energy distribution characteristics of different positions are different [9]. Since the three-point bending test cannot reflect the internal energy state of structure under uniform load conditions, the uneven energy input and the energy concentration make concrete producing the fracture energy, and the peak of the energy and its distribution position affect the fracture form of the concrete beam [10-11].

For the damage and fracture of the loaded concrete beams, Liao et al. studied the shear resistance and damage characteristics of high-strength reinforced concrete beams under dynamic loads using physical model tests, and they evaluated the damage of beam at different loading stages [12]. Based on the theory of elastoplastic mechanics, Betschoga et al. conducted the model test to investigate the influence of boundary conditions and loading modes on shear resistance of concrete, and they studied the mechanical mechanism of crack development of the beam structures under different loading modes [13]. Almaliki et al. analyzed the fracture process of the concrete beams and they discussed the influence of flexural mechanical parameters on the fracture mode [14]. There is a large crack development zone in the process of concrete beam fracture, the existing research mostly focuses on the fracture toughness of critical instability moment or the energy consumption of complete fracture moment [15-16], however, the research on the energy consumption in the fracture process zone accompanying cracks development is relatively little. Based on the energy balance principle, Hoque et al. predicted the bending failure form of concrete beams and they found that the propagation laws of defects crack of the beam are determined by the releaseable strain energy of the concrete. More attention should be paid to the relationship between the accumulated strain energy and the dissipated energy in the initiation stage, stable expansion stage and instability expansion stage of the rupture zone of the concrete beam [17].

The essence of beam failure is the instability of the state driven by various external energies [18]. In the process of the concrete damage, the development and penetration of microscopic defects such as cracks and pores are accompanied by energy dissipation [19]. For concrete beam structures under external load, the existing research focuses on the deformation, the displacement and the fracture characteristics of the beam structure, and rarely involves the energy accumulation, release and dissipation of the loaded concrete beams. Both theoretical analysis and experiments show that the energy dissipation of the concrete beams has nonlinear characteristics in the process of the plastic deformation and fracture [20-23], and the storage and the release of strain energy have an important influence on structural equilibrium state and fracture behavior of the beam [24].

In this study, according to stress-strain relationship of the concrete material, the energy accumulation and dissipation characteristics of the concrete units and the calculation method were analyzed. To explore the stability of the equilibrium state of the structural system and the characteristics of the accumulation and release of energy during bending deformation, the mechanical model of the simple supported concrete beam under uniform loading was constructed and the corresponding numerical model was built by using FLAC ${ }^{3 \mathrm{D}}$. The characteristics of the peak strain energy, residual elastic energy and dissipated energy of the loaded concrete units were monitored and analyzed.

The rest of this study is organized as follows. Section 3 describes the establishment of the mechanical model and the research methods. Section 4 gives the results and discussion, and finally, the conclusions are summarized in Section 5.

\section{Methodology}

\subsection{Mechanical model of accumulation and release of energy}

The concrete beam is treated as a non-tension beam in unit thickness. As shown in Fig. 1(a), the self-weight load of the concrete beam is simplified as $q$ of the uniformly distributed load. According to its force boundary conditions, the concrete beam can be regarded as the mechanical model shown in Fig. 1(b). $L$ is the span of the beam. $y$ is the value of bending sinking deformation of the concrete beam under the action of $q$ of the vertical uniform load. Eq. (1) can be used to describe its sinking curve shape.

$$
y=f \sin \frac{\pi x}{L}
$$

where, $x$ is the distance from the origin $O$ to any point $K$ on the beam. $y$ is the bending sinking amount corresponding to $x$. $f$ is the bending sinking amount of mid-point $O$ of the simply supported concrete beam .

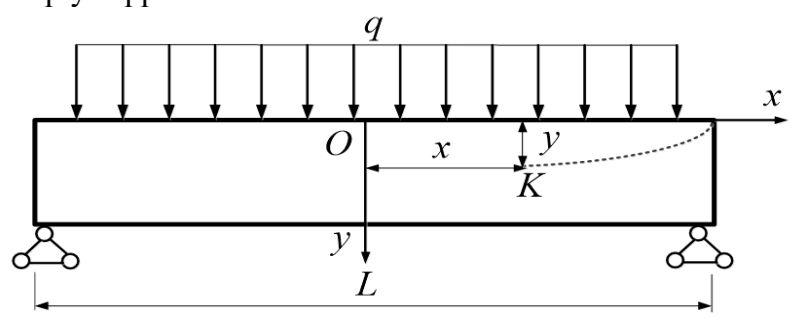

(a) Before bending.

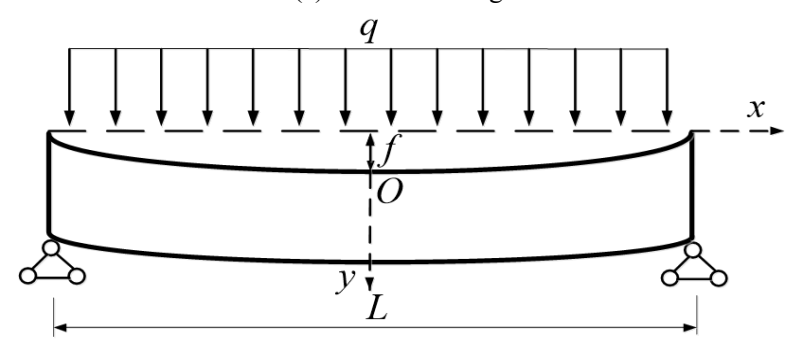

(b) After bending.

Fig. 1. Simply supported concrete beam under uniform load.

Since the bending failure of the concrete beam is the instability of state driven by energy, based on the change of elastic potential energy in the process of the deformation of the concrete beam, the stability of the concrete structure can be analyzed. The total strain energy $U$ is composed of the structural strain energy $U_{l}$ and the external load potential energy which is generated by the uniform load $q$. The strain energy $U_{l}$ accumulated by the concrete beam structure due to deformation is 
$U_{1}=\frac{E I}{2} \int_{-\frac{L}{2}}^{\frac{L}{2}} t^{2} d x$

where, $I$ is the inertia moment of the cross section. $E$ is the elasticity modulus. $t$ is the curvature of any point $x$.

\subsection{Numerical simulation of accumulation and release of} energy in loaded concrete beam

Concrete materials are always exchanging energy with the external environment in the process of deformation destruction. The samples in a laboratory have the energy exchange with the testing machine. Considering the concrete unit, its deformation under the action of external force is regarded as an adiabatic physical process. The concrete deformation failure is mainly determined by the releasable elastic energy $U^{e}$ and the damage dissipation energy $U^{d}$. If the work of the testing machine is $W$, then the total input energy $U$ of the concrete sample is equal to $W$. According to the energy conservation law, the energy relationship is as follows [1-2] :

$$
U=U^{\mathrm{e}}+U^{\mathrm{d}}
$$

Loading of quasi-brittle materials such as concrete and rock are always accompanied by energy storage and consumption in the process of deformation failure. The energy dissipation is unidirectional and irreversible, but under certain conditions the energy release is bidirectional and reversible [3-6]. According to the loading and unloading curves at each level, the unloading elastic modulus, the releasable strain energy, and the dissipated energy of the concrete material can be measured, as shown in Fig. 2.

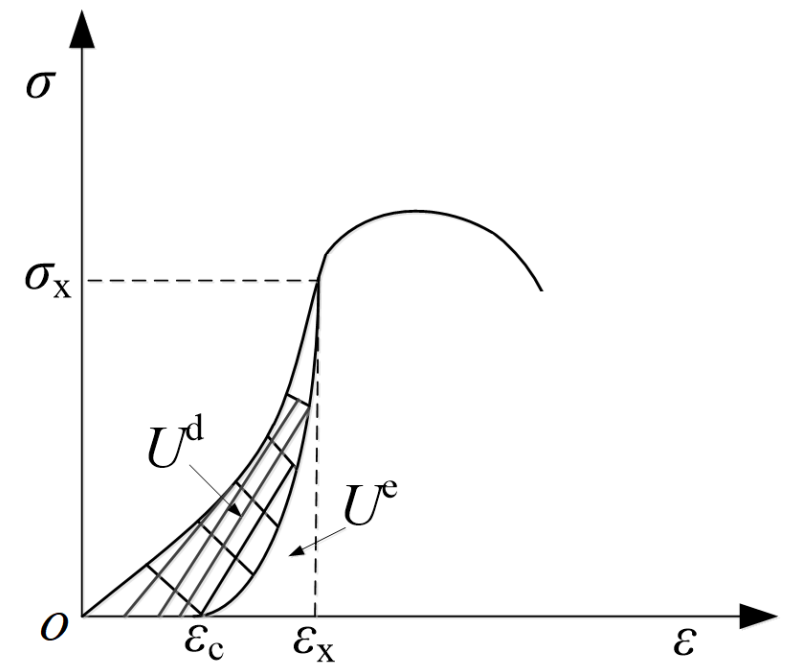

Fig. 2. Relationship between the dissipated energy and the elastic energy in concrete units.

According to the stress-strain curve of the loading and unloading, if the unloading is performed when the load reaches $\sigma_{x}$, the area of the loading curve from zero to $\varepsilon_{x}$ represents the total input energy $U$ of the concrete unit. When the stress is loaded to $\sigma_{x}$, the area of the unloading curve the $\varepsilon_{c}$ strain to the $\varepsilon_{x}$ strain is $U^{e}$ of the releasable elastic energy stored in the sample. After unloading, the strain energy of this part will be released. The area from the loading to the unloading curves represents $U^{d}$ of the consumed energy during the plastic deformation and damage of the concrete unit, namely
$U^{e}=\int_{\varepsilon_{c}}^{\varepsilon_{x}} \sigma d \varepsilon$

$U^{d}=\int_{0}^{\varepsilon_{c}} \sigma d \varepsilon$

$U=U^{e}+U^{d}=\int_{0}^{\varepsilon_{x}} \sigma d \varepsilon$

where, the unit of $U, U^{e}$ and $U^{d}$ is $\mathrm{MJ} / \mathrm{m}^{3}$, and it represents the energy level of per unit volume concrete.

The indices of stress and strain can be used to describe the mechanical state of the concrete beam structures, but it cannot fully reflect its thermodynamic state. The elastic strain energy is a comprehensive index, and in this index the structural stress and deformation are involved simultaneously. Under the action of uniformly distributed load, the concrete beam structure releases energy within a certain range and causes the change of strain energy state. The failure process of the loaded concrete beam system is a comprehensive result of the accumulation and release of strain energy. Energy dissipation occurs gradually with the increase of the load in the concrete structures, and the deterioration of concrete mechanical properties and the strength decrease are always accompanied by the energy dissipation.

Assuming that there are $n$ units in the concrete system, the strain energy density $K$ of unit body depends on the three principal stresses and principal strains of the space, and the calculation formula is as follows [18]:

$$
\begin{aligned}
K & =\frac{1}{2}\left(\sigma_{1} \varepsilon_{1}+\sigma_{2} \varepsilon_{2}+\sigma_{3} \varepsilon_{3}\right) \\
& =\frac{1}{2 E}\left[\sigma_{1}^{2}+\sigma_{2}^{2}+\sigma_{3}^{2}-2 \mu\left(\sigma_{1} \sigma_{2}+\sigma_{2} \sigma_{3}+\sigma_{1} \sigma_{3}\right)\right]
\end{aligned}
$$

where, $\sigma_{i}(i=1,2,3)$ are three principal stresses of a certain unit body. $\varepsilon_{i}(i=1,2,3)$ are three principal strains of a certain unit body.

The strain energy $U_{i}$ of the $i$-th unit body is:

$U_{i}=\int K_{i} d V_{i}$

where, $K_{i}$ is the strain energy density of the $i$-th unit. $V_{i}$ is the volume of the $i$-th unit.

Then $U$ of the total strain energy of the concrete beam structure is

$U=\sum_{i=1}^{n} U_{i}$

Then, the dissipated energy of the concrete beam structure can be expressed as

$U_{0}^{\mathrm{d}}=W-\sum_{i=1}^{n} U_{i}$

As shown in Fig. 3, the computational model of the simply supported concrete beam and the supporting platform were established by FLAC ${ }^{3 \mathrm{D}}$ software. The cross-section size of the concrete beam is $400 \mathrm{~mm}$ wide and $400 \mathrm{~mm}$ high, and the length is $4000 \mathrm{~mm}$. The supporting platforms at both ends of beam are regarded as elastic supports. The concrete 
beam and the supporting platform are respectively selected from Mohr-Coulomb model and the elastic model, and the interface contact surfaces are set between the supporting platform and the concrete beam to simulate the hinged contact and separation process after deformation. The model is divided into 2000 units and 2946 nodes.

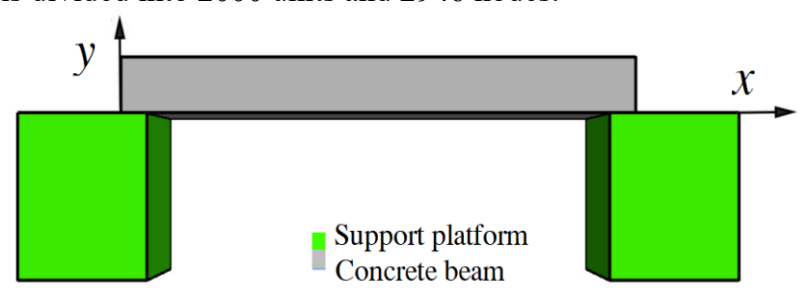

Fig. 3. Numerical model of simply supported concrete beam.

The uniformly distributed load 0.5 MPa was applied to the upper surface of the concrete beam in the process of loading. According to Eqs. (5), (7) and (8), the built-in fish language programming of FLAC ${ }^{3 \mathrm{D}}$ was used to calculate the elastic strain energy and dissipation energy of the concrete unit. The horizontal displacement constraints are imposed on both sides of the support platforms and the vertical displacement constraints are imposed on its bottom. The concrete strength of $\mathrm{C} 30$ is set. The parameters of the concrete beam are shown in Table 1, and the elastic modulus and Poisson's ratio of the supporting platforms are the same as the beam.

Table 1. Material parameters in the numerical model.

\begin{tabular}{c|c|c|c|c|c}
\hline $\begin{array}{c}\text { Weight } \\
\left(\mathbf{k N} / \mathbf{m}^{\mathbf{3}}\right)\end{array}$ & $\begin{array}{c}\text { Elastic } \\
\text { modulus } \\
\mathbf{( G P a )}\end{array}$ & $\begin{array}{c}\text { Poisson's } \\
\text { ratio }\end{array}$ & $\begin{array}{c}\text { Cohesion } \\
\mathbf{( M P a )}\end{array}$ & $\begin{array}{c}\text { Internal } \\
\text { friction } \\
\text { angle }\left(^{\circ}\right)\end{array}$ & $\begin{array}{c}\text { Tensile } \\
\text { strength } \\
(\mathbf{M P a})\end{array}$ \\
\hline 2400 & 33 & 0.16 & 3.18 & 55 & 1.80 \\
\hline
\end{tabular}

\section{Results and discussion}

\subsection{Accumulation and release analysis of energy}

In the model of the simply supported concrete beam, the work done by the uniform load $q$ is:

$W_{q}=\int_{-\frac{L}{2}}^{\frac{L}{2}} q f \sin \frac{\pi x}{L} d x$

The dissipated energy of the simply supported concrete beam structures due to deformation failure under uniformly distributed loads is:

$U_{1}^{\mathrm{d}}=\int_{-\frac{L}{2}}^{\frac{L}{2}} q f \sin \frac{\pi x}{L} d x-\frac{E I}{2} \int_{-\frac{L}{2}}^{\frac{L}{2}}\left(\frac{d^{2} y}{d x^{2}}\right)^{2}\left(1-\left(\frac{d y}{d x}\right)^{2}\right)^{-1} d x$

Eq. (12) is expanded by Talor, and the high-order traces are omitted, the integral is calculated as:

$U_{1}^{d}=\frac{2 q L f}{\pi}-\frac{E I \pi^{6}}{16 L^{5}} f^{4}-\frac{E I \pi^{4}}{4 L^{3}} f^{2}$

The first and second partial derivatives of the dissipated energy $U_{1}^{d}$ with respect to $f$ are respectively:

$$
\begin{aligned}
& \frac{\partial U_{1}^{d}}{\partial f}=\frac{2 q L}{\pi}-\frac{E I \pi^{6}}{4 L^{5}} f^{3}-\frac{E I \pi^{4}}{2 L^{3}} f \\
& \frac{\partial^{2} U_{1}^{d}}{\partial f^{2}}=-\frac{3 E I \pi^{6}}{4 L^{5}} f^{2}-\frac{E I \pi^{4}}{2 L^{3}}
\end{aligned}
$$

It can be seen that $\frac{\partial^{2} U_{1}^{d}}{\partial f^{2}}$ is less than zero from Eq. (15), therefore, the total strain energy of the simply supported concrete beam obtains the maximum value. The beam structure is unstable during the gradual subsidence deformation, and the equilibrium configuration cannot be maintained. When the system reaches the bifurcation point along the stable equilibrium path, the tensile or shear failure of the concrete beams will undergo, and the structural dissipation energy will display a sudden increase.

\subsection{Simulation analysis of accumulation and release of energy}

The deformation of bending sinking of the concrete beam under uniform loading of $0.5 \mathrm{MPa}$ is shown in Fig. 4. The vertical sinking amount of the mid-span position is the largest, and the vertical displacement gradually decreases from the mid-span to two ends of the beam, and the peak of vertical displacement is $31.9 \mathrm{~mm}$. The elastic strain energy distribution of the simply supported concrete beam after bending shows the bridge type distribution characteristics. The concentration of strain energy in the mid-span upper surface of the beam is the highest, and the peak value of elastic strain energy can reach $4517.5 \mathrm{~J}$. The accumulated energy is transferred from the mid-span to the support platforms of both ends in the beam structure. The strain energy is released due to bending deformation of lower part of the beam, and the strain energy concentration at both ends of the beam is small.

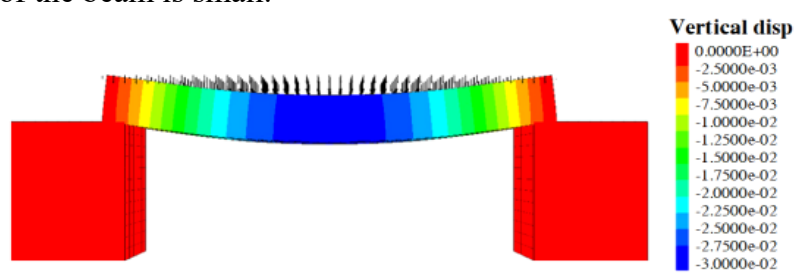

(a) Bending sinking features of the beam.

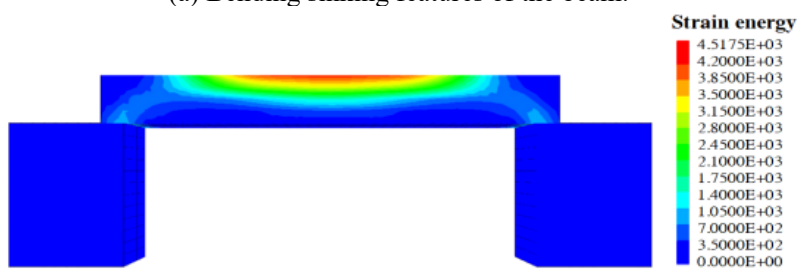

(b) Distribution characteristics of elastic energy.

Fig. 4. Strain energy distribution characteristics of the simply supported concrete beam in bending.

As shown in Fig. 5, the lower zone of the simply supported concrete beam produces shear-tensile failure due to bending deformation under the uniform load. The failure zone of the beam concentrates in the release zon e of strain energy, and no failure units appear in the mid-span strain energy concentration zone. The concrete units at the position of the support platforms produce shear failure. There are tension-shear mixed failure units in the empty surface area at the lower part of the beam. The failure zone of the simply supported concrete beam has obvious energy dissipation occurrence. Due to the tension and shear effects of the lower part unit of the beam, high-values of the dissipation energy are distributed near the support platforms, and the peak value of the dissipation energy is $3532.3 \mathrm{~J}$. 


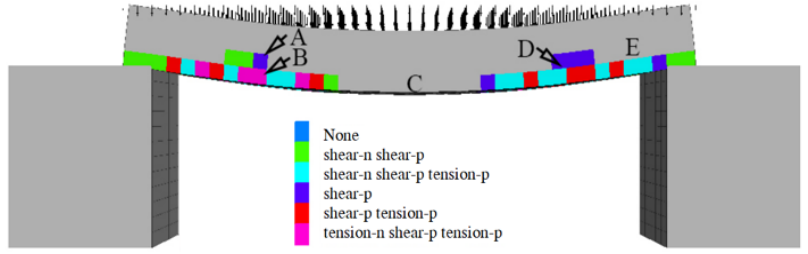

(a) Deformation failure of the concrete beam.

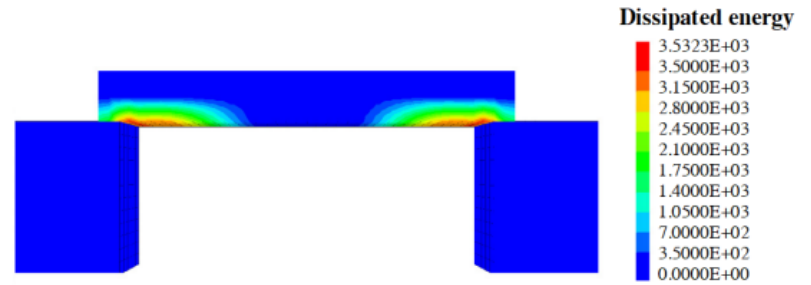

(b) Energy dissipation characteristics of the concrete beam. Fig. 5. Energy dissipation characteristics of the concrete beams after failure.

According to the monitoring data of the failure units of A, B, D and the undamaged units of $\mathrm{C}$ and $\mathrm{E}$ in Fig. 5(a), the strain energy change characteristics of these units in the process of loading are obtained. As shown in Fig. 6(a), the strain energy of the concrete units gradually increases at the beginning of loading, there is the peak in the elastic energy of the failure units $\mathrm{A}$ and $\mathrm{B}$, and the peak of the elastic energy of the lower unit $B$ is ahead of that of the upper unit A, while the peak of the elastic energy of the upper unit $A$ is higher than that of the lower unit B. After the strain energy reaches its peak, the strain energy of the units begins to be released. After the energy being released, there is still a certain amount of the residual strain energy in the unit.

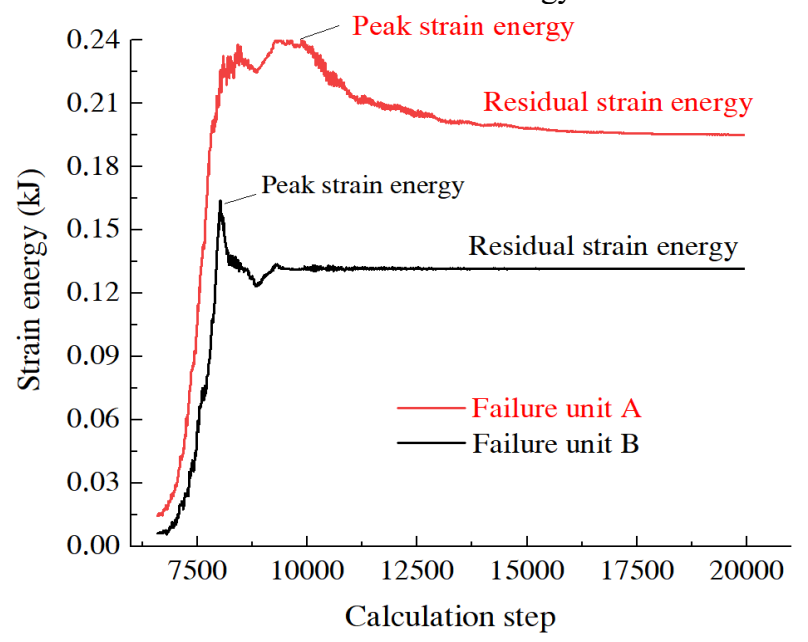

(a) Units A and B.

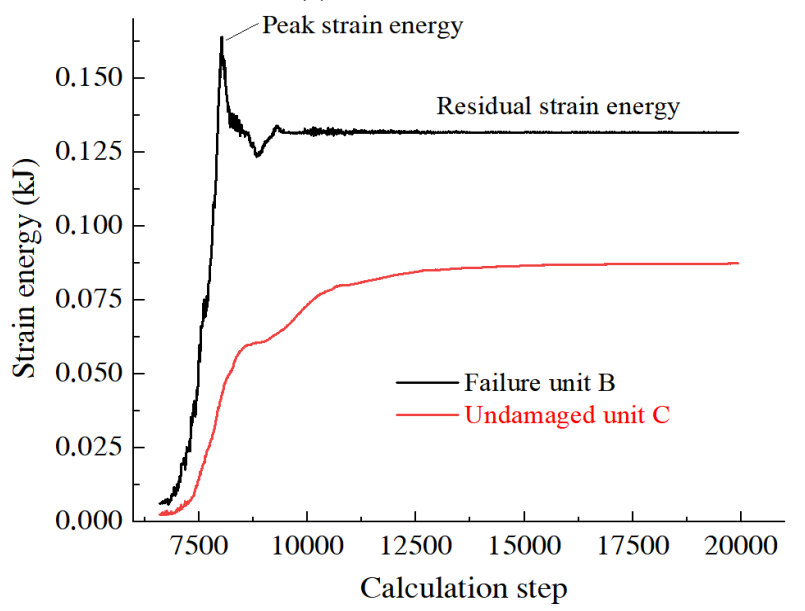

(b) Units B and C.

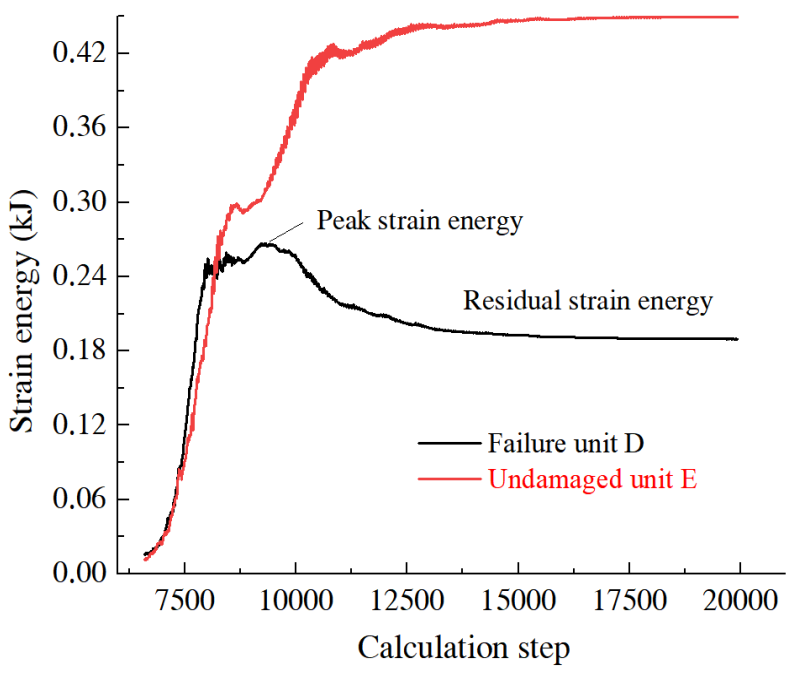

(c) Units D and E.

Fig. 6. Curves of the elastic strain energy of the monitoring units in the model.

The elastic energy characteristics of the failure unit B on the left lower side of the beam and the undamaged unit $\mathrm{C}$ on the mid-span bottom are shown in Fig. 6 (b). The strain energy of the unit $\mathrm{B}$ is overall higher than that of the unit $\mathrm{C}$, and the elastic energy of the undamaged unit $\mathrm{C}$ does not release after reaching the peak. The elastic energy change characteristics of the failure unit D and the undamaged unit $\mathrm{E}$ on the right lower side of the beam are shown in Fig. 6(c). The unit E near the support platform is subjected to the concentrated stress and large deformation, and its elastic energy level is higher than that of the failure unit D.

\subsection{Energy dissipation analysis of the loaded concrete beam in the fracture process}

Fig. 7 shows the characteristics of the dissipated energy of the failure units $\mathrm{A}$ and $\mathrm{B}$ of the concrete beam and undamaged unit $\mathrm{C}$. The lower unit $\mathrm{B}$ with tensile-shear failure has a significantly higher dissipation energy than the upper unit A with simple shear failure. The unit of the midspan position is undamaged, and the dissipated energy of the undamaged unit $\mathrm{C}$ on the mid-span position is zero $\mathrm{J}$. It can be seen that due to the plastic deformation and damage of the loaded concrete unit, the failure units generate energy dissipation, and they have complex failure forms, and their dissipation energy are higher than that of the simple shear failure units.

The concrete unit undergoes an energy accumulation process before fracture. As shown in Fig. 8, the compressive stress and deformation of the unit $\mathrm{A}$ in the pre-peak elastic state gradually increase, and the strain energy of the unit continues to rise, and no energy dissipation occurs in the process. When the stress exceeds the elastic limit, the unit body produces the plastic deformation and dissipated energy. After that, the dissipated energy curve shows a nonlinear upward trend. After the unit body reaching the energy storage limit, it fractures and produces a sudden energy release, as a result its dissipation energy curve under the condition of a small displacement manifests a sharp rise.

There are some differences between the energy accumulation and the displacement at different positions of the concrete beam in the horizontal direction in the process of the flexural deformation. As seen from Fig. 9(a), the curves show the relationships between strain energy peak of the fracture zone and the vertical displacement of the 
concrete beam. Along the horizontal direction, the maximum value of the strain energy of each unit is located at the inflection point of curvature, and in this position the unit first reaches the energy storage limit and fractures. The relationships between the residual strain energy and displacement of each unit body are shown in Fig. 9(b). The energy peak value and residual value of the mid-span position are lower than that of the other positions. In the process of rupture, the peak zone of the storage energy produces larger energy release, as a result, the peak value of the residual strain energy after ruptures exists at the boundary position of both sides of the beam.

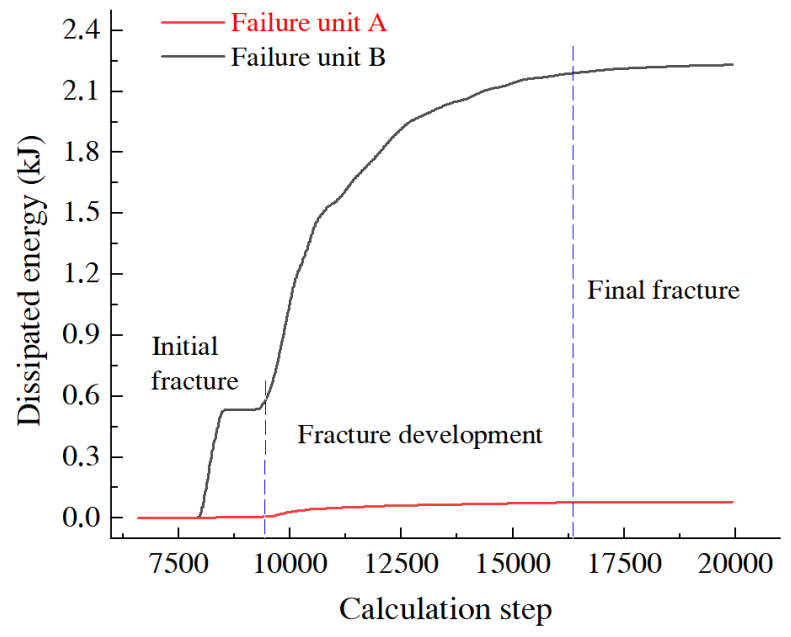

(a) Units A and B.

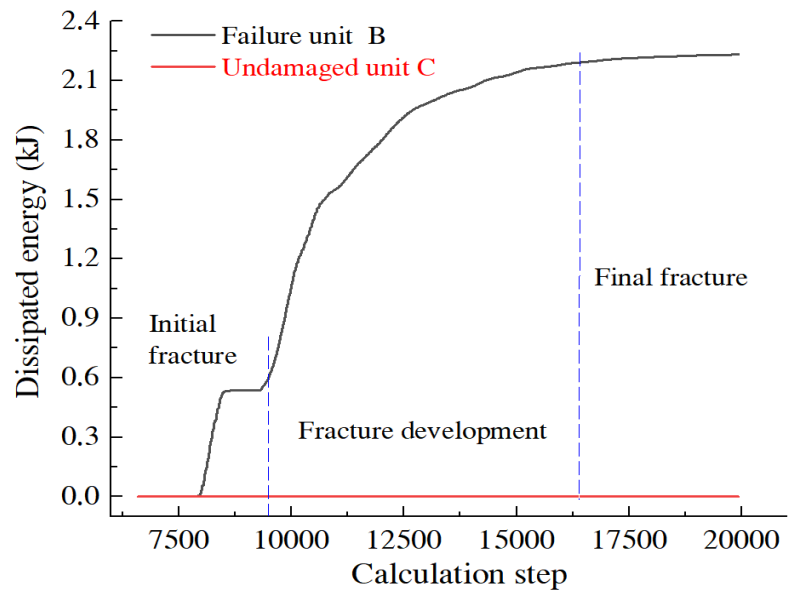

(b) Units B and C.

Fig. 7. The dissipated energy characteristics of the monitoring units in the model.

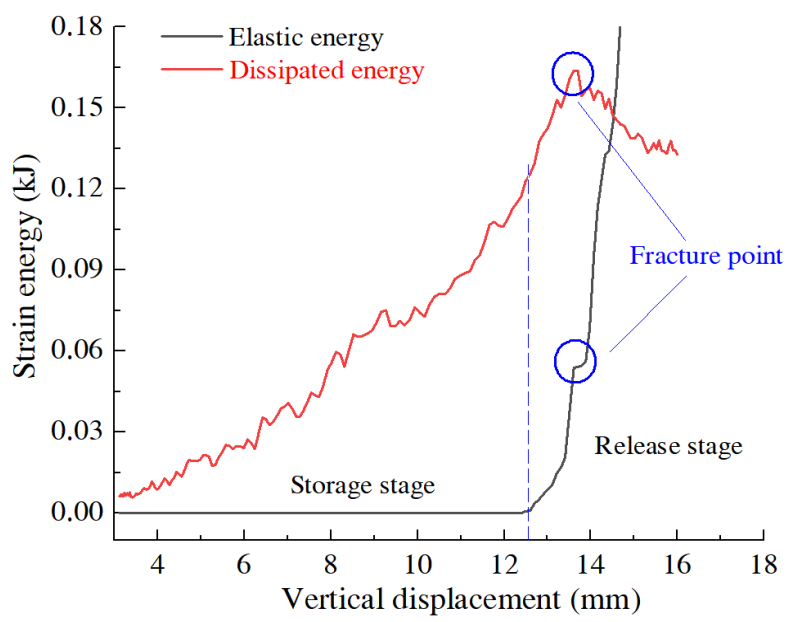

Fig. 8. Energy accumulation and dissipation characteristics before and after fracture of the unit A.

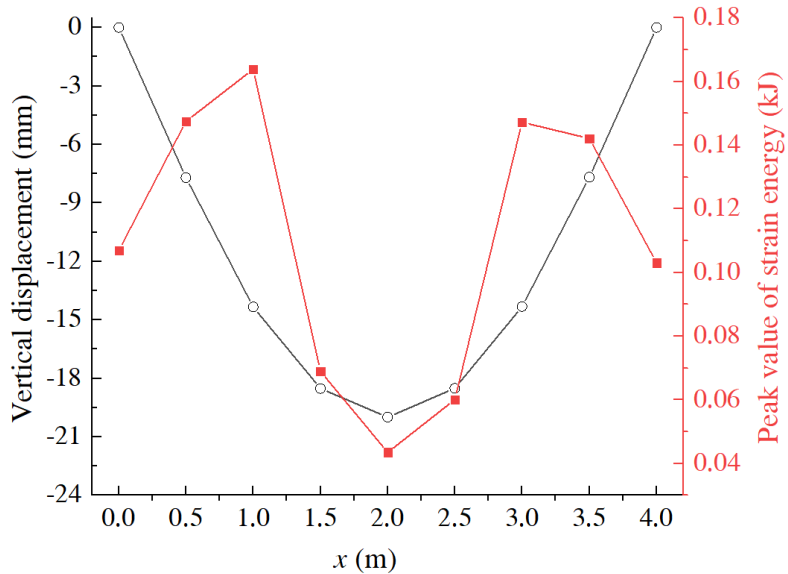

(a) Peak strain energy and vertical displacement.

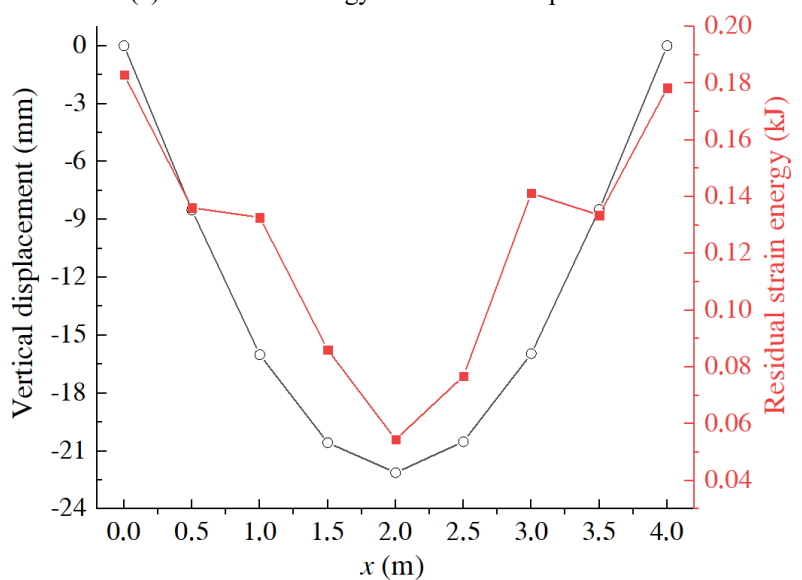

(b) Residual strain energy and vertical displacement.

Fig. 9. Relationship curves between the strain energy and vertical displacement of the fracture zone.

According to the monitoring data of the strain energy and dissipated energy of the monitoring unit of each layer of the concrete beam in Fig. 10, the peak value of the elastic energy gradually increases from bottom to top along thickness direction of the beam under the action of the uniformly distributed load. The peak value of the elastic energy of the upper part of beam is the highest, and the peak value of the elastic energy of the upper part of the mid-span is higher than that of both sides. Because the failure units of the concrete beam are mainly concentrated in the empty surface of the beam bottom, the dissipated energy of this position is significantly higher than that of other positions of the beam.

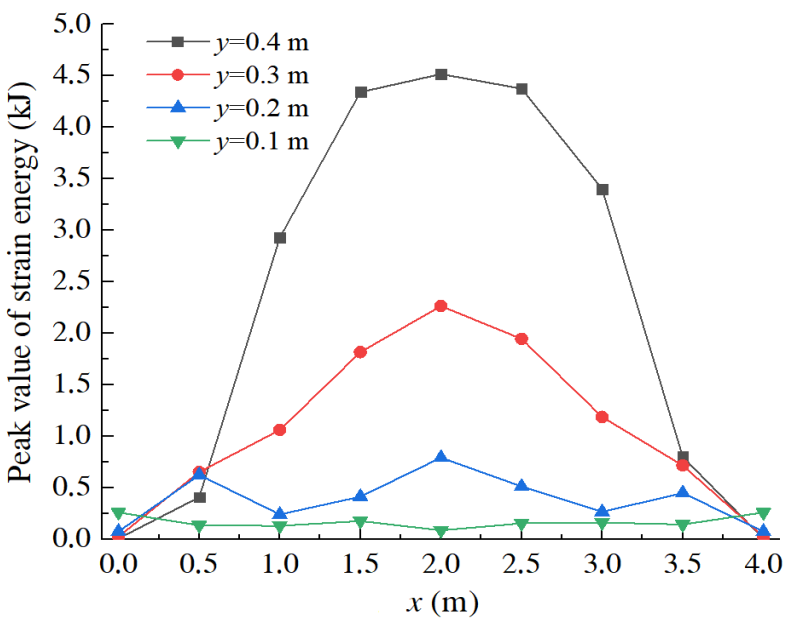

(a) Peak values of elastic energy of different layers. 


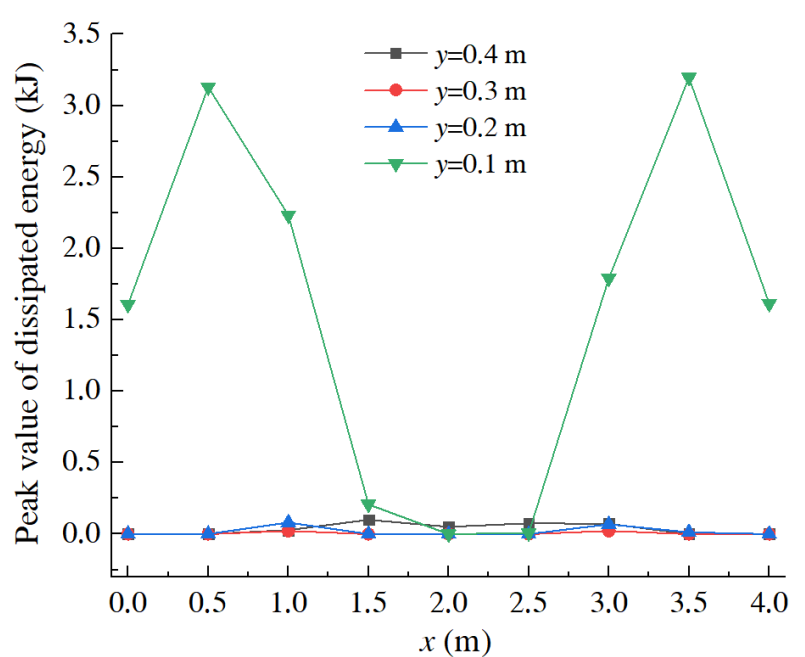

(b) Peak values of dissipated energy of different layers.

Fig. 10. Peak values of elastic energy and dissipated energy of different layers of the concrete beam.

\section{Conclusions}

To study energy evolution characteristics of the simply supported concrete beams in the process of bending fracture under the uniformly distributed load, the structural mechanical and numerical models were constructed, and the strain energy distribution characteristics of the beam structure at different deformation stages were analyzed based on the principle of energy balance. The influence of the energy accumulation and release on the rupture form was discussed also. The differences of the energy dissipation behaviors of different positions in the process of rupture were summarized. The main conclusions are obtained as follows:

(1) The strain energy of the simply supported concrete beam after bending under the uniformly distributed load has the bridge distribution characteristics. The strain energy concentration of the upper region of the mid-span of the beam is significantly higher than that of the two sides, and the peak of the elastic energy exists at the upper surface of the mid-span of the beam. The failure units of the simply supported concrete beams are concentrated in the energy release zone. Undamaged units appear in the energy concentration zone in the mid-span of the beam. The concrete beam units at the support platforms produce shear failure. There are tension-shear mixed failures in the empty surface of the beam bottom units, and the obvious energy dissipation phenomenon appears in the failure area.

(2) The strain energy of the concrete beams continues to rise in the initial stage of loading. The elastic energy of the concrete unit releases and causes material damage after reaching its peak. The residual elastic energy of the concrete unit still exists after failure. The elastic energy of the undamaged unit of the mid-span is lower than that of the failure units of the same layer of both sides. The peak value of the elastic energy gradually increases from the bottom to top along the thickness direction of the beam. The peak value of the elastic energy of the upper part of the beam is the highest, and the peak value of the elastic energy of the upper part of the mid-span is higher than that of the both sides.

(3) The failure units of the simply supported concrete beams are concentrated in the strain energy release zone under the uniform loading. There is obvious energy dissipation in the failure areas. The dissipation energy of the concrete units with tensile-shear failure is higher than that of the simple shear failure units. The dissipated energy of the bottom of the beam is significantly higher than the dissipated energy of other layers.

This study mainly focuses on the flexural deformation of the simply supported beams under the uniformly loading, the energy distribution and dissipation characteristics obtained are symmetrical, but there are also inhomogeneities in the external loads in the actual engineering, and the skew characteristics of the asymmetric deformation and the energy evolution cannot be reflected. In the next work, the deformation law of the concrete beam under the action of the concentrated loading or non-uniform loading will be further studied.

\section{Acknowledgements}

This work was financially supported by the National Natural Science Foundation of China (51774112), and the Fundamental Research Funds for the Universities of Henan Province (NSFRF200202), China.

This is an Open Access article distributed under the terms of the Creative Commons Attribution License.

\section{References}

1. Carpinteri, A., Lacidogna, G., Corrado, M., Battista, E. D., "Cracking and crackling in concrete-like materials: A dynamic energy balance". Engineering Fracture Mechanics, 155, 2016, pp. 130-144

2. Shadman, M., Ziari, H., "Laboratory evaluation of fatigue life characteristics of polymer modified porous asphalt: A dissipated energy approach". Construction \& Building Materials, 138, 2017, pp. 434-440.

3. Wang, S. R., Xiao, H. G., Hagan, P., Zou, Z. S. "Mechanical behavior of fully-grouted bolt in jointed rocks subjected to double shear tests". Dyna, 92(3), 2017, pp. 314-320.

4. Jarosińska, M., Abramowicz, M. Berczyński, S., "Experimental validation of the use of energy transfer ratio (ETR) for damage diagnosis of steel-concrete composite beams". Journal of Theoretical and Applied Mechanics, 55(1), 2017, pp. 241-252.

5. Zhao, Y. H., Wang, S. R., Zou, Z. S., Ge, L. L., Cui, F. "Instability characteristics of the cracked roof rock beam under shallow mining conditions". International Journal of Mining Science and Technology, 28(3), 2018, pp. 437-444.
6. Tung, N. D., Tue, N. V., "Effect of support condition and load arrangement on the shear response of reinforced concrete beams without transverse reinforcement". Engineering Structures, 111, 2016, pp. 370-382.

7. Ghahremannejad, M., Abolmaali, A., "Prediction of shear strength of reinforced concrete beams using displacement control finite element analysis". Engineering Structures, 169, 2018, pp. 226-237.

8. Pham, A. T., Tan, K. H., "Static and dynamic responses of reinforced concrete structures under sudden column removal scenario subjected to distributed loading". Journal of Structural Engineering, 145(1), 2019, pp. 04018235.

9. Mrozinski, S., "Energy-based method of fatigue damage cumulation". International Journal of Fatigue, 121, 2019, pp. 73-83.

10. Haq, M., Bhalla, S., Naqvi, T., "Fatigue damage monitoring of reinforced concrete frames using wavelet transform energy of pztbased admittance signals". Measurement, 164, 2020, pp. 108033. 
11. Lemery, J., Ftima, M. B., Leclerc, M., Wang, C., "The disturbed fracture process zone theory for the assessment of the asymptotic fracture energy of concrete". Engineering Fracture Mechanics, 231(2), 2020, pp. 107022.

12. Liao, Z., Tang, D., Li, Z. Z., Xue, Y. L., Shao, L.Z., "Study on explosion resistance performance experiment and damage assessment model of high-strength reinforcement concrete beams". International Journal of Impact Engineering, 133, 2019, pp. 103362.

13. Betschoga, C., Tung. N. D., Tue, N. V., "Investigations on the influence of boundary and loading conditions on the shear resistance of FRP concrete beams without shear reinforcement". Composite Structures, 262, 2021, pp. 113335.

14. Almaliki, H. N. G., Al-Balhawi, A., Alshimmeri, A. J. H., Zhang, B. S., "Structural efficiency of hollow reinforced concrete beams subjected to partial uniformly distributed loading". Buildings, 11(9), 2021, pp. 1-16.

15. Liu, B. B., Shi, Q. Y., Yang, F., "Energy characteristics of acoustic emission signals of the reinforced concrete beams during bending failure and the auxiliary location". Science Technology and Engineering, 15(6), 2015, pp. 246-249.

16. Li, Z. Y., Li, Y. X., Yao, Z. H., Xu, X., Wu, Y. F., "Mechanical damage model for concrete based on energy dissipation". China Civil Engineering Journal, 52(S1), 2019, pp. 24-30.

17. Hoque, N., Jumaat, M. Z., Sulong, N. H. R., "Global energy balance-based debonding modeling of NSM FRP-strengthened concrete beam". Journal of Composites for Construction, 24(1), 2020, pp. 04019053.
18. Yang, H., Feng, Y., Wang, H. X., Jeremic, B., "Energy dissipation analysis for inelastic reinforced concrete and steel beam-columns". Engineering Structures, 197, 2019, pp. 109431.

19. Guo, J. H., Cai, J., Chen, Q. J., Liu, X. P., Wang, Y. Q., Zuo, Z. L., "Dynamic behaviour and energy dissipation of reinforced recycled aggregate concrete beams under impact". Construction and Building Materials, 214, 2019, pp. 143-157.

20. Achintha, M., Burgoyne, C., "Fracture Energy of the Concrete FRP Interface". Engineering Fracture Mechanics, 110, 2013, pp. 38-51.

21. Ključanin, D., Manđuka, A. "The cantilever beams analysis by the means of the first-order shear deformation and the Euler-Bernoulli theory". Technical Journal, 13(1), 2019, pp. 63-67.

22. Wang, S. R., Xu, D. F., Hagan, P., Li, C. L. "Fracture characteristics analysis of double-layer rock plates with both ends fixed condition". Journal of Engineering Science and Technology Review, 7(2), 2014, pp. 60-65.

23. Yu, R. Q., Zhang, D. D., Yan, H. C., "Embodied energy and cost optimization of RC beam under blast load". Mathematical Problems in Engineering, 2017, 2017, pp. 1907972.

24. Lacidogna, G., Piana, G., P., Accornero, F., Carpinteri, A., "Multitechnique damage monitoring of concrete beams: acoustic emission, digital image correlation, dynamic identification". Construction and Building Materials, 242, 2020, pp. 118114. 